Research Article

\title{
Knowledge and Compliance Toward Standard Precaution Among Health Care Workers at Bahria International Hospital Lahore, Pakistan
}

\author{
Humira Ather, Asaf Khan, Saima Shabnum*
}

Bahria International Hospital, Lahore, Pakistan

\section{Article Information \\ Received: 07 December 2020 \\ Revised version received: 20 January 2020 \\ Accepted: 22 January 2020 \\ Published: 27 January 2020 \\ Cite this article as: \\ H. Ather et al. (2020) Int. J. Soc. Sc. Manage. 7(1): 17-20. DOI: $\underline{10.3126 / \mathrm{ijssm} . v 7 \mathrm{i} 1.27406}$}

\section{*Corresponding author \\ Humira Ather, \\ Bahria International Hospital, Lahore, Pakistan \\ Email: saima.shabnum1278@gmail.com}

Peer reviewed under authority of IJSSM

(C) 2020 International Journal of Social Sciences and Management

\section{OPEN AACCESS}

This is an open access article \& it is licensed under a Creative Commons Attribution Non-Commercial 4.0 International (https://creativecommons.org/licenses/by-nc/4.0/)

Keywords: Knowledge; Practice; Standard Precautions; Nurse

\begin{abstract}
Introduction: Standard precaution is a way to stop the spread of hospital acquired infection which may be in the form of blood, secretions, body waste, body fluids and mucous membrane that may contain contagious infectious agents. Healthcare workers are the persons who have the moral obligation to care for sick persons and improve their regaining health and attain excellent worth of treatment care. Therefore, health care providers should have proper knowledge and good practice to strictly adhere to standard precaution control infection. Aim: To determine the knowledge and compliance toward standard precaution among health care workers in Bahria international Hospital Lahore Methodology: a descriptive cross sectional study design were used for this study with sample size of 180 participants which were randomly selected from the target population Results: The association between knowledge and practice was assessed through chi square test with $\mathrm{p}$ value $=0.05$, after apply this test the $\mathrm{p}$ value found .000 which is significant association between knowledge and practice. Whereas regression test shows value of adjusted $\mathrm{R}^{2}$ showing $60 \%$ $(\mathrm{F}=.141, \mathrm{p}<.001)$ of variance caused by independent variable (knowledge) in dependent variable (practice). Hence, it is proved that there is a significant positive relationship between knowledge and practice. Conclusion: The current study concluded that adhering to standard precaution is very important for all health care workers to control hospital acquired infection. Study finding also show that majority of the healthcare workers have good knowledge regarding standard precautions but they have unsatisfactory compliance to prevent and control infection.
\end{abstract}

\section{Introduction}

Health care workers are constantly exposed to microorganisms. Many of which can cause serious or even fatal infections (Kosgeroglu, et al., 2004; National Health and Medical Research Council, 2010). The finding of one study showed that out 3 million Health care workers experience exposure to blood born viruses. The results is estimated 16,000 hepatitis C, 66,000 hepatitis B, and 5000 immunodeficiency virus(HIV) infections annually and more than $90 \%$ of the infections occurred in low income countries which can be prevented Both the healthcare workers and patients in a healthcare setting are at risk of contracting an infection (WHO, 2011) However, in clinical setting the Doctors and Nurses are continuously exposed while they are taking care of patient. Therefore, the risk becomes high if Nurses and Doctors do not have knowledge and compliance of standard precaution (SP) (Wilburn and Eijkman, 2004). Furthermore, literature is evident that by using of simple techniques of standard precaution has been 
shown to reduce the risk of exposure to blood and body fluids (Siegel, 2007). The main crust of standard precaution demonstrating by knowledge, practice and compliance of, hand hygiene, personal protective equipment (PPE), waste management, linen management, patient care equipment, prevention of needle stick injuries and the safe discarding of sharps. (Nagliate et al., 2013).

According to one of Iran study results revealed proper knowledge and practice of the Standard precautions can considerably decrease the incidence of occupational exposure amongst Nurses (Kermode et al., 2005). Similarly, a study testing knowledge of the Standard precautions shows that only $57.1 \%$ of nursing and midwifery Ethiopia had 'sufficient knowledge (Lawoyin et al., 2006). A study is affirmed that about $21 \%$ nurses, and $30 \%$ paramedics were unaware that Hepatitis B and C can be transmitted with needle stick injuries. In addition, Gammon \& Gould (2005) reported that health care workers have limited knowledge and training on standard precaution. On contrary, a Pakistani study showed that only $4.7 \%$ of physicians reported to cleanse their hands before having direct contact with their patients.

In study some of responded had a needle stick injuries in past but out of all only $7 \%$ reported and rest did not. Further, $27 \%$ used gloves for drawing patient blood and 295 felt that needles could be recapped after use (Alam, 2002). Furthermore, Sigel, affirms this assertion, that with hand washing, personal hygiene becomes the fundamental principle in observing standard precautions. (WHO. 2002). Moreover, another study showed that $50 \%$ of Nurses and $41 \%$ of Doctors did not wear gloves during blood exposure accident (Lawoyin et al., 2006). In north India study revealed that a high proportion of health care workers were not complying needle recapping precaution. Some of studies showed the reasons for noncompliance of Standard precautions are lack of knowledge, lack of time, lack of administrative support, heavy work load (Kolude et al., 2004)

\section{Purpose of the Study}

The purpose of this study is to assess knowledge and practice of health care worker regarding standard precautions.

\section{Problem Statement}

It is observed that most health care worker are not following the standard precautions. Health care worker are at high risk of exposure of infectious diseases if they did not adapt standard precautions protocol and they also transmit the infectious agents to the patients. Health care worker should have appropriate knowledge, attitude and practice toward standard precautions by which they can decrease the morbidity and mortality rate and ultimately enhance patient health quality.
According to Baqi et al. (2009), he concluded a study it is revealed from his study that health care workers in government hospitals were not following the standard precautions protocols. (Baqi, 2009). Health care worker have poor knowledge and practices toward standard precautions and this lead to increase infection rate (Mangoni, 2012). Health care worker appropriate knowledge and refine practices toward standard precautions play a vital role in prevention of hospital acquired infections and increase patient outcome (Eskander et al., 2013).

\section{Significance of the Study}

Health care worker have close contact to the patients, therefore results of the study will be helpful for Health care worker to follow the standard precautions to minimize hospital acquired infections. It will also help the participants of the study to become aware about the adherence of standard precautions and its consequences for them and for patients. After completion of this study results will be provided to organization to know the importance of using standard precautions. The finding of this study will enable the policy makers and higher authority of the hospital to build infection control committee to improve the Health care worker practices and attitude and enhance their knowledge to improve patient care and incorporate the standard isolation precaution in the hospital.

\section{Materials and Methods}

\section{Study Population}

The study participants for this study were all the health care workers of Bahria international hospital which include Doctors, Nurses, LHVs, LPNs, Nursing assistant, Technicians.

\section{Study Setting}

Setting for this study were different wards of hospital, Doctors OPD and Nursing Counters of Bahria international Hospital.

\section{Study Design}

A cross sectional study design was used to conduct this study.

\section{Data Collection Procedure}

A standardized, structured self-administered questionnaire were developed after a thorough literature search. The questionnaire has three main sections:

\section{Section 1: Biographical Data}

This section is comprised questions on biographical data (age and gender), area of practice. Qualification, Clinical Experience and a question regarding exposure to any needle stick Injury, the purpose of eliciting such information was to secure a descriptive profile of respondents and to ensure a basis for data analysis in relation to other sections of the questionnaire as per the objectives of the study. Closeended questions were used to collect the biographical data. 


\section{Section 2: Knowledge of Standard Precautions}

This section contained questions which sought to ascertain the level of knowledge and understanding of the concept of standard precautions including post-exposure prophylaxis. Multiple-choice questions were used to assess the knowledge of standard precautions among study participants.

\section{Section 3: Practice of standard Precautions and factors that influence compliance to Standard precautions}

Section 3 comprised questions on the level of adherence to standard precautions. These sections were also including questions on the use and availability of personal protective equipments, vaccination and policy and guidelines for SP.

After questionnaire developed it will be sent to 3 experts in infection control to check for face and content validity and Cronbach's alpha will be calculated. After revision, the questionnaire will be piloted on a group of nurses and if there is need the necessary modification will be made. A random sample of 10 nurses will be taken and questioner will be run on them to establish the ability of the tool to achieve the stated study objectives and determine the logistics, such as time taken to complete the clinical observation checklist and questionnaire. The participants and findings from the pilot test were excluded from the main study.

\section{Data Collection Method}

Opportunity were given to all healthcare workers from different departments to participate in this study, the researcher explained the purpose of the study and that participation in the study were voluntary. A written consent was being taken from participants detailing the purpose of the study, confidentiality and protection of the data, contact information for the PI, and assurances about the protection of the anonymity of responses. This was a 12-item questionnaire with both open-ended and closed questions

in English; it took 10-15 minutes to complete.

The participants will be instructed that do not talk to each other while they are filling the form.

\section{Data Analysis}

The data from the questionnaire were coded and entered into a Microsoft Excel spreadsheet and analysis were done using SPSS, version 21. For quantities variables in descriptive variables: mean and standard deviation were calculated. Chi square and linear Regression test were applied for the analysis of data.

\section{Ethical Consideration}

To proceed with proposed study official permissions letter were obtained from the ethical committee of Bahria international Hospital. Prior to initial interview, researchers introduced themselves to study participants, explain the purpose of study, and ensure that privacy and confidentiality.

\section{Sampling Technique}

Simple random sampling techniques were used in this study for collecting participants' response.

\section{Results and Discussions}

It includes 3 portions of analysis. First analysis was demographic analysis. It gives us details of four demographic questions. Descriptive analysis was used for 3 variables. One was independent variables (Factor affecting practice) and two were dependent variables (Knowledge and Practice). It tells us knowledge and practice of doctors and nurses and the factors that affecting their practices. Chi-Square test analysis is used for the current study. Hospital acquired infection is a major problem in all over the world. Thus, refine knowledge and polished practice toward standard precaution can play important roles in preventing infection. Healthcare workers should have to practice according to standard precaution protocols on daily basis as an essential part of patients' care to prevent infection. That is why this cross sectional study was carried out. Standard precautions are central actions for preventing hospital assimilated infections and act as protective measures for health care providers from infection through professional contact. Finding of the study indicated that if the nurses have good knowledge about standard isolation precaution then they positively affect their practice but a little change can also occur in behavior.

The relationship between knowledge and practice is positive and relationship is significant. So the knowledge is very important that affects the practice of performance significantly. The present study reveals that knowledge of the participants regarding standard precautions was satisfactory $71 \%$ of the study participants knows the definition of standard precaution, $53 \%$ of the study participants were agreed and knows that healthcare workers are the common source of hospital acquired infection while $28 \%$ stated the HCWs have no role in hospital acquired infection while $19 \%$ were not sure

These findings are also supported by study Baqi et al. (2009) which revealed that the majority (91.6\%) of the study participants had awareness about standard isolation precautions of prevention of infection. 97\% knew that standard precautions should be accomplished on all patients. When inquired about recommendations for management of sharps instrument $47.7 \%$ told that sharps should be recapped. The most of the participants $(95.8 \%)$ have knowledge that before after direct contact with patient's hands should be washed while $96.5 \%$ aware that when draw blood sample gloves should be wear. $73 \%$ have good knowledge that when touching a patient's surroundings hand hygiene should be done.

The present study also stated that nurse's practices were poor according to CDC guideline of preventing hospital acquired infection. The current study reveals that $28.9 \%$ of 
the participants always wash their hand before and after patient care, $8.9 \%$ often, $35.6 \%$ sometime and mostly $26.7 \%$ were never wash their hand before and after patient care. Responses of the participants to another that wearing of gloves is necessary when drawing blood sample in which $48.3 \%$ participants were response to always, $18.3 \%$ often, $15.0 \%$ were sometime and $3318.3 \%$ were never wear gloves while taking blood sample which show poor practice (CDC, 2001).

According to study Mangoni (2012) which shows about the practice of nurses, hand hygiene should done, $58.5 \%, 28.1 \%$ and $63.6 \%$ always practiced hand hygiene after touching patients, after touching patients' surroundings and after removing gloves, respectively which show that nurses practice are not satisfactory and they must have to improve their clinical skill or practice. Only twenty-two per cent always wear gloves before taking blood sample of patient which also shows the poor practice of nurses. In relation to injection safety, $33.7 \%$ usually recap sharps with two hands, $7.9 \%$ sometimes bend or break sharps, while $63.6 \%$ had always disposed of sharps/needles in puncture proof boxes.

\section{Conclusion}

The current study concluded that using standard precaution protocol is necessary for all healthcare workers to prevent hospital acquired infection. Study finding also show that most of the participants in the study have good knowledge about standard precaution protocols but there practice were not satisfactory toward standard precaution. Study finding also predict that participants have poor practice and they do not follow proper standard precaution protocol to prevent infection. The study also concluded that there is positive association between knowledge and practice which means that if the level of knowledge increase than practice will be improve if the environment of hospital is free of hazards.

\section{References}

Alam M (2002) Knowledge, attitude and practices among health care workers on needle-stick injuries. Annals of Saudi Medicine 22(5-6): 395.

Baqi S, Damani NN, Shah SA, Khanani R (2009) Infection control at a government hospital in Pakistan. Int J Infect Control 5(1): 1-7.

CDC (2001) Updated U.S. Public Health Service guidelines for the management of occupational exposures to $\mathrm{HBV}, \mathrm{HCV}$, and HIV and recommendations for post exposure prophylaxis. MMWR 50: $1-42$.
Eskander HG, Morsy WYM \& Elfeky HAA (2013) Intensive Care Nurses' Knowledge \& Practices regarding Infection Control Standard Precautions at a Selected Egyptian Cancer Hospital. Prevention 4(19): 160-174.

Gammon J \& Gould D (2005) Universal precautions: A review of knowledge, compliance and strategies to improve practice. Journal of Research in Nursing 10(5): 529-547. https://doi.org/10.1177/136140960501000503

Kermode M, Jolley D, Langkham B, Thomas MS, Holmes W \& Gifford SM 2005 Compliance with universal/standard precautions among health care workers in rural north India. American Journal of Infection Control 33(1): 2733.

Kolude OO, Omokhodion FO and Owoaje ET (2004) Universal Precaution: Knowledge, Compliance and perceived risk of infection among doctors at a University Teaching Hospital. Int. conf. AIDS; Abstract no MoPed 3676.

Kosgeroglu N, Ayranci U, Vardareli E \& Dincer S (2004) Occupational exposure to hepatitis infection among Turkish nurses: frequency of needle exposure, sharps injuries and vaccination. Epidemiol Infect 132(1): 27-33.

Lawoyin T, Stringer B, Taines and Oluwatosin A (2006) Assessing the risk of the health care workers for occupational transmission of HIV, hepatitis B and C in Ibadan, Nigeria. 145 Conf HIV patho treatment.

Mangoni (2012) The first Global Patient safety challenge: "Clean care is safer care". Geniva. International Journal of Nursing Education 48(2): 45-56.

Nagliate P. Nogueira P, Godoy S and Merdes I (2013) Measures of .Knowledge About Standard Precaution: A Literature Review. Nurse Education in Practice 13: 244-249.

National Health and Medical Research Council (2010) Australian Guidelines for the Prevention and Control of Infection in Healthcare Canberra.

Siegel JD, Rhinehart E, Jackson M \& Chiarello L (2007) Healthcare Infection Control Practices Advisory Committee. Guideline for isolation precautions: preventing transmission of infectious agents in healthcare settings. Centers for Disease Control

WHO (2002) Reducing Risk, Promoting Health Life. World Health Report. Geneva.

WHO (2011) Report on the burden of endemic health careassociated infection worldwide. Edited by Allegranzi B. Geneva: World Health Organisation.

Wilburn SQ \& Eijkemans G (2004) Preventing needle stick injury among health care workers: a WHO-ICN collaboration. Int J Occup Environ Health 10(4): 451. 